\title{
Demanda hídrica da cana-de-açúcar irrigada por gotejamento nos tabuleiros costeiros de Alagoas
}

\author{
Samuel Silva ${ }^{1}$, José Dantas Neto ${ }^{2}$, Iêdo Teodoro ${ }^{3}$, José L. de Souza ${ }^{3}$, \\ Guilherme B. Lyra ${ }^{3} \&$ Marcos A. L. dos Santos ${ }^{4}$ \\ ${ }^{1}$ Programa de Pós-Graduação em Engenharia Agrícola/Universidade Federal de Campina Grande. Campina Grande, PB. E-mail: sam_capela@hotmail.com \\ (Autor correspondente) \\ ${ }^{2}$ Unidade Acadêmica de Engenharia Agrícola/Centro de Tecnologia e Recursos Naturais/Universidade Federal de Campina Grande. Campina Grande, \\ PB. E-mail: zedantas1955@gmail.com \\ ${ }^{3}$ Centro de Ciências Agrárias/Universidade Federal de Alagoas. Rio Largo, AL. E-mail: iedoteodoro@ig.com.br; leonaldojs@yahoo.com.br; \\ gbastoslyra@yahoo.com.br \\ ${ }^{4}$ Departamento de Engenharia Rural/Faculdade de Ciências Agronômicas/Universidade Estadual Paulista "Júlio de Mesquita Filho". Botucatu, SP. E-mail: \\ liodorio@hotmail.com
}

\section{Palavras-chave:}

Saccharum spp.

armazenamento de água no solo déficit hídrico

\begin{abstract}
R E S U M O
A água é um recurso natural que, nos últimos anos, tem sido alvo de inúmeras discussões quanto ao seu uso e disponibilidade. No setor agrícola o volume de água necessário à irrigação é calculado com base nas perdas ocorridas por evaporação e transpiração motivo por que este trabalho teve, como objetivo, determinar a evapotranspiração máxima e real da cana-de-açúcar irrigada por gotejamento. Os dados para esta pesquisa foram obtidos em um experimento conduzido na região de Rio Largo, AL, com sete tratamentos de irrigação $\left(0 ; 25 ; 50 ; 75 ; 100 ; 125\right.$ e 150\% da evapotranspiração de referência-ET $\left.{ }_{0}\right)$, em três ciclos de produção no período de janeiro de 2009 a fevereiro de 2012. A evapotranspiração real $\left(\mathrm{ET}_{\mathrm{r}}\right)$ foi estimada pelo $\mathrm{K}_{\mathrm{c}}$ "dual". A ET $\mathrm{r}$ média na fase de crescimento máximo dos três ciclos variou de 1,73 a $5,04 \mathrm{~mm} \mathrm{~d}^{-1}$ nos tratamentos com 0 e $150 \%$ da $\mathrm{ET}_{0}$, respectivamente. A cana-de-açúcar em regime de sequeiro está, nesta região, exposta a médio risco climático, conforme classificação proposta na literatura.
\end{abstract}

Key words:

Saccharum spp. water storage in the soil moisture deficit

\section{Water requirement of drip irrigated sugarcane in the Coastal Lands of Alagoas}

\footnotetext{
A B S T R A C T

Water is a natural resource that has been the subject of much debate about its use and availability in recent years. In the agricultural sector, the volume of water needed for irrigation is calculated based on the losses incurred by evaporation and transpiration. Thus, this study aimed to determine the maximum and actual evapotranspiration of drip irrigated sugarcane. The data for this study were obtained in an experiment conducted in the region of Rio Largo (Alagoas, Brazil), with seven irrigation treatments $(0 ; 25 ; 50 ; 75$; $100 ; 125$ and $150 \%$ of the reference evapotranspiration - $\left.\mathrm{ET}_{0}\right)$, in three production cycles from January 2009 to February 2012. The actual evapotranspiration $\left(\mathrm{ET}_{\mathrm{r}}\right.$ ) was estimated by "dual" $\mathrm{K}_{c}$. The mean $\mathrm{ET}_{\mathrm{r}}$ in the maximum growth phase of the three cycles ranged from 1.73 at $5.04 \mathrm{~mm} \mathrm{~d}^{-1}$ in the treatments with 0 and $150 \%$ of $\mathrm{ET}_{0}$, respectively. The sugarcane, in rainfed conditions in this region, is exposed to average climatic risk, according to the classification proposed in the literature.
} 


\section{INTRODUÇÃo}

A cana-de-açúcar é uma cultura de alto valor econômico no Brasil e no mundo devido à sua utilização na produção de açúcar, álcool e energia elétrica, em que um dos fatores que mais afetam a sua produtividade agroindustrial é o déficit hídrico causado pela irregularidade de chuvas em algumas regiões brasileiras, dentre as quais se destaca a região Nordeste (Silva et al., 2014).

O Estado de Alagoas é o maior produtor de cana-deaçúcar do Nordeste, ou seja, a cultura de maior expressão socioeconômica regional porém, mesmo possuindo elevado nível tecnológico no setor sucroalcooleiro há, nesta região, problemas que afetam a produção de cana-de-açúcar destacando-se a distribuição irregular de chuvas durante o período anual. Embora a média pluviométrica anual seja de $1.800 \mathrm{~mm}$, cerca de $70 \%$ das chuvas se concentram no período de abril a agosto enquanto $30 \%$ se distribuem de outubro a fevereiro (Souza et al., 2004; Carvalho et al., 2013b). Esta falta de uniformidade na distribuição da precipitação pluvial no decorrer do ano resulta em menor armazenamento de água no solo durante o período seco favorecendo redução na evapotranspiração real, no crescimento e na produtividade da cultura; desta forma, é imprescindível o uso de irrigação nesta região (Dantas Neto et al., 2006; Teodoro et al., 2009).

Estudos relacionados ao consumo de água pela cultura e o uso de recursos hídricos para a irrigação vem-se tornando cada vez mais frequentes de vez que tal operação é um dos fatores de maior influência na produtividade agrícola e no custo de produção da cana-de-açúcar (Teodoro et al., 2013). Para suprir as necessidades hídricas das plantas deve-se estimar a evapotranspiração da cultura (ET). Assim, para que as plantas atinjam o potencial evapotranspiração é necessário que o teor de umidade do solo esteja igual ou próximo da capacidade de armazenamento de água disponível (CAD). No entanto, devido à presença de pragas e doenças, salinidade, baixa fertilidade do solo e escassez ou saturação de água, a $\mathrm{ET}_{c}$ se torna diferente do valor máximo obtido em condições ótimas, sendo chamada de evapotranspiração real (ET) (Pereira et al., 2002; Allen et al., 2005).

O requerimento hídrico total da cana-soca irrigada, variedade RB92579, cultivada por aproximadamente 12 meses na região semiárida do Submédio do Vale do São Francisco, é de $1.710 \mathrm{~mm}$ (Silva et al., 2012b). Almeida et al. (2008) mediram, porém, a $\mathrm{ET}_{\mathrm{c}}$ acumulada de $1.584 \mathrm{~mm}$ durante 12 meses, em Rio Largo, AL, para a mesma variedade (RB92579) e no segundo ciclo de cultivo irrigado. Lyra et al. (2007a) estimaram a $\mathrm{ET}_{\mathrm{r}}$ total de $1.180 \mathrm{~mm}$ durante 15 meses para a cana-planta cultivada no regime de sequeiro na região de Maceió, AL, utilizando o método do K "dual”; no entanto, ao adotar o $\mathrm{K}_{\mathrm{c}}$ conjugado para a evaporação e transpiração na mesma época de cultivo Lyra et al. (2007b) estimaram a ET total de $2.050 \mathrm{~mm}$; esses resultados indicam que o método "dual" considera separadamente a evaporação da água presente no solo, o que diminui o valor total de ETr sendo, a partir de então, mais indicado para estimavas de evapotranspiração de culturas irrigadas por gotejamento.

Tendo em vista a grande demanda de água durante o ciclo de cultivo da cana-de-açúcar, sistemas de irrigação mais eficientes no uso da água, como o de gotejamento, são imprescindíveis (Parkes et al., 2010; Boas et al., 2011; Martins et al., 2011). Por isto já existe tendência de expansão de áreas irrigadas por gotejamento por parte dos produtores alagoanos visando à substituição da irrigação por aspersão mesmo que ainda existam dúvidas com relação ao volume de água economicamente viável a ser aplicado por meio da irrigação por gotejamento.

Considerando o aumento expressivo do uso de gotejamento nos canaviais irrigados de Alagoas objetivou-se, com o presente trabalho, determinar a evapotranspiração real da cultura da cana-de-açúcar irrigada por gotejamento, sob lâminas de irrigação diferenciadas, durante três ciclos de produção nos Tabuleiros Costeiros de Alagoas.

\section{Material e Métodos}

O experimento foi conduzido a partir do cultivo da canade-açúcar em uma área de 0,5 ha, no Centro de Ciências Agrárias da Universidade Federal de Alagoas, em Rio Largo, AL (09² $\left.28^{\prime} 02^{\prime \prime} \mathrm{S} ; 35^{\circ} 49^{\prime} 43^{\prime \prime} \mathrm{O} ; 127 \mathrm{~m}\right)$. O período experimental compreendeu três ciclos de produção da cultura, desde janeiro de 2009 a fevereiro de 2012. O delineamento estatístico utilizado foi o de blocos casualizados com sete tratamentos e quatro repetições. As parcelas foram constituídas de 10 linhas com $12 \mathrm{~m}$ de comprimento e os tratamentos constaram de sete lâminas de irrigação por gotejamento nos níveis de 0 (L0), 25 (L1), 50 (L2), 75 (L3), 100 (L4), 125 (L5) e 150\% (L6) da evapotranspiração de referência $\left(\mathrm{ET}_{0}\right)$.

O solo foi classificado por Carvalho (2003) como Latossolo Amarelo coeso argissólico de textura média/argilosa. As características físicas e topográficas são especificadas como: densidade volumétrica (ds) de $1,5 \mathrm{Mg} \mathrm{m}^{-3}$, porosidade total (P) de $0,423 \mathrm{~m}^{3} \mathrm{~m}^{-3}$, velocidade de infiltração básica (VIB) de 52 $\mathrm{mm} \mathrm{h}^{-1}$ e declividade média menor que $2 \%$. Este solo, quando mantido na capacidade de campo (CC) e no ponto de murcha permanente (PMP), possui valores de umidade de 0,2445 e $0,1475 \mathrm{~m}^{3} \mathrm{~m}^{-3}$, respectivamente. O clima é caracterizado pela classificação de Thornthwaite e Mather, como quente e úmido $\left(B_{1}\right)$, megatérmico $\left(A^{\prime}\right)$, com deficiência de água moderada no verão ( $\mathrm{s}$ ) e grande excesso de água no inverno $\left(\mathrm{w}_{2}\right)$. A precipitação pluvial média da região é de $1.800 \mathrm{~mm}$ (Souza et al., 2004).

As variáveis agrometeorológicas foram disponibilizadas pelo Laboratório de Agrometeorologia e Radiometria Solar (LARAS), o qual mantém uma estação automática de aquisição de dados (Modelo Micrologger CR10X, Campbell Scientific, Logan, UT, EUA) instalada a cerca de $400 \mathrm{~m}$ do experimento. Os procedimentos de cálculo de evapotranspiração real da cultura $\left(\mathrm{ET}_{\mathrm{r}}\right)$ e balanço de água no solo, usados para a estimativa do $\mathrm{K}_{\mathrm{c}}$ "dual", foram baseados na metodologia descrita por Allen et al. (2005) e adaptados por Silva et al. (2012a) para a cana-de-açúcar irrigada por gotejamento.

A evapotranspiração de referência $\left(\mathrm{ET}_{0}\right)$ foi calculada pelo método de Penman - Monteith (Allen et al., 2005). A evapotranspiração real da cultura $\left(\mathrm{ET}_{\mathrm{r}}\right)$ foi calculada pelo método "dual", em que o coeficiente da cultura $\left(\mathrm{K}_{\mathrm{c}}\right)$ é dividido 
em dois coeficientes: um para a transpiração da cultura $\left(\mathrm{K}_{\mathrm{cb}}\right)$ e outro para a evaporação do solo $\left(\mathrm{K}_{\mathrm{e}}\right)$, conforme apresentado na Eq. 1. O coeficiente $K_{s}$ representa os efeitos do déficit de água no solo na zona radicular sobre a $\mathrm{ET}_{\mathrm{r}}$.

$$
\mathrm{ET}_{\mathrm{r}}=\mathrm{K}_{\mathrm{s}} \times \mathrm{ET}_{\mathrm{c}}=\mathrm{K}_{\mathrm{s}} \times \mathrm{K}_{\mathrm{c}} \times \mathrm{ET}_{0}=\left(\mathrm{K}_{\mathrm{s}} \times \mathrm{K}_{\mathrm{cb}}+\mathrm{K}_{\mathrm{e}}\right) \times \mathrm{ET}_{0}
$$

O armazenamento de água no sistema radicular da cultura no dia i $\left(\mathrm{ARM}_{\mathrm{i}}\right)$ foi calculado em função do balanço de água no solo no dia i (Eq. 2), considerando o armazenamento do dia anterior e o limite do solo em armazenar água (CAD). Para dar início ao cálculo do balanço de água no solo foi considerado que o ARM era igual a CAD na data de plantio ( $1^{\circ}$ ciclo de produção) e no corte da cana-de-açúcar $\left(2^{\circ}\right.$ e $3^{\circ}$ ciclos de produção) pois foi aplicada uma irrigação nessas datas com tal finalidade.

$$
\mathrm{ARM}_{\mathrm{i}}= \begin{cases}\max \left(\mathrm{ARM}_{\mathrm{i}-1}+\mathrm{ALT}_{\mathrm{i}}, 0\right) ; & \text { para } \rightarrow \mathrm{ARM}_{\mathrm{i}-1}+\mathrm{ALT}_{\mathrm{i}}<0 \\ \max \left(\mathrm{ARM}_{\mathrm{i}-1}+\mathrm{ALT}_{\mathrm{i}}, \mathrm{CAD}\right) ; & \text { para } \rightarrow \mathrm{ARM}_{\mathrm{i}-1}+\mathrm{ALT}_{\mathrm{i}}>0\end{cases}
$$

em que:

$\mathrm{ARM}_{\mathrm{i}}$ - armazenamento de água na zona radicular ao término do dia $\mathrm{i}, \mathrm{mm}$;

$\mathrm{ARM}_{\mathrm{i}-1}$ - conteúdo de água na zona radicular ao término do dia anterior, mm;

$\mathrm{ALT}_{\mathrm{i}}$ - alteração do armazenamento da água do solo no dia i calculada como $\mathrm{P}_{\mathrm{i}}+\mathrm{I}_{\mathrm{i}}-\mathrm{ET}_{\mathrm{r}, \mathrm{i}}, \mathrm{mm}$;

$\mathrm{P}_{\mathrm{i}} \quad$ - precipitação no dia $\mathrm{i}, \mathrm{mm}$;

$I_{i} \quad$ - lâmina de irrigação líquida no dia i, mm; e

$\mathrm{ET}_{\mathrm{r}, \mathrm{i}}$ - evapotranspiração real da cultura no dia $\mathrm{i}, \mathrm{mm}$ ).

Finalmente, foram calculados o índice de satisfação das necessidades de água (ISNA) para o tratamento de sequeiro em todo o ciclo de produção e a evapotranspiração relativa da cultura (ER) no período seco para os tratamentos irrigados, ambos definidos como a razão entre a Evapotranspiração real $\left(E_{r}\right)$ e a Evapotranspiração máxima $\left(E T_{c}\right)$ da cultura (Silva et al., 2013), sendo que a última fase do ciclo de produção da cultura não foi utilizada para este fim visto que o déficit hídrico provocado nesta fase foi necessário para induzir as plantas à maturação.

\section{Resultados e Discussão}

A precipitação pluvial durante o $1^{\circ}$ ciclo de produção da cultura (12/01/2009 a 22/02/2010 - 406 dias) somou $2.208 \mathrm{~mm}$, mas $63 \%$ (1.384 $\mathrm{mm}$ ) deste valor ocorreram entre os meses de abril e agosto de 2009 (Figura 1). O mês de maio de 2009 foi o mais chuvoso, no total de $454 \mathrm{~mm}$.

No $2^{\circ}$ ciclo de produção (23/02/2010 a 28/02/2011 - 371 dias), a precipitação acumulada foi $1.952 \mathrm{~mm}$, porém 1.180 $\mathrm{mm}$ correspondentes a $60 \%$ do valor total foram registrados durante a estação chuvosa (de abril a agosto de 2010). O mês de junho de 2010 foi o mais chuvoso de todo o período analisado, com $579 \mathrm{~mm}$, sendo que no dia 4 deste mês foram registrados $186 \mathrm{~mm}$ (quase $10 \%$ do total no ciclo). No $3^{\circ}$ ciclo de produção, referente ao período compreendido de 29/02/2011 a 20/02/2012 - 357 dias, o total precipitado foi $1.915 \mathrm{~mm}$ sendo $75 \%$ observados entre abril e agosto de 2011 . Neste ciclo o mês de abril foi o mais chuvoso, com $404 \mathrm{~mm}$. Considerando os três ciclos de cultivo, $66 \%$ da precipitação ocorreram, em média, entre os meses de abril e agosto, caracterizando uma distribuição irregular ao longo do ano.

Conforme Souza et al. (2004), na zona canavieira alagoana a precipitação pluvial normal é de $1.300 \mathrm{~mm}$ ao longo do ano enquanto na região de Rio Largo a média anual é de $1.800 \mathrm{~mm}$, ou seja, aproximadamente $70 \%$ das chuvas se concentram no período de abril a agosto (estação chuvosa). Assim, há grande

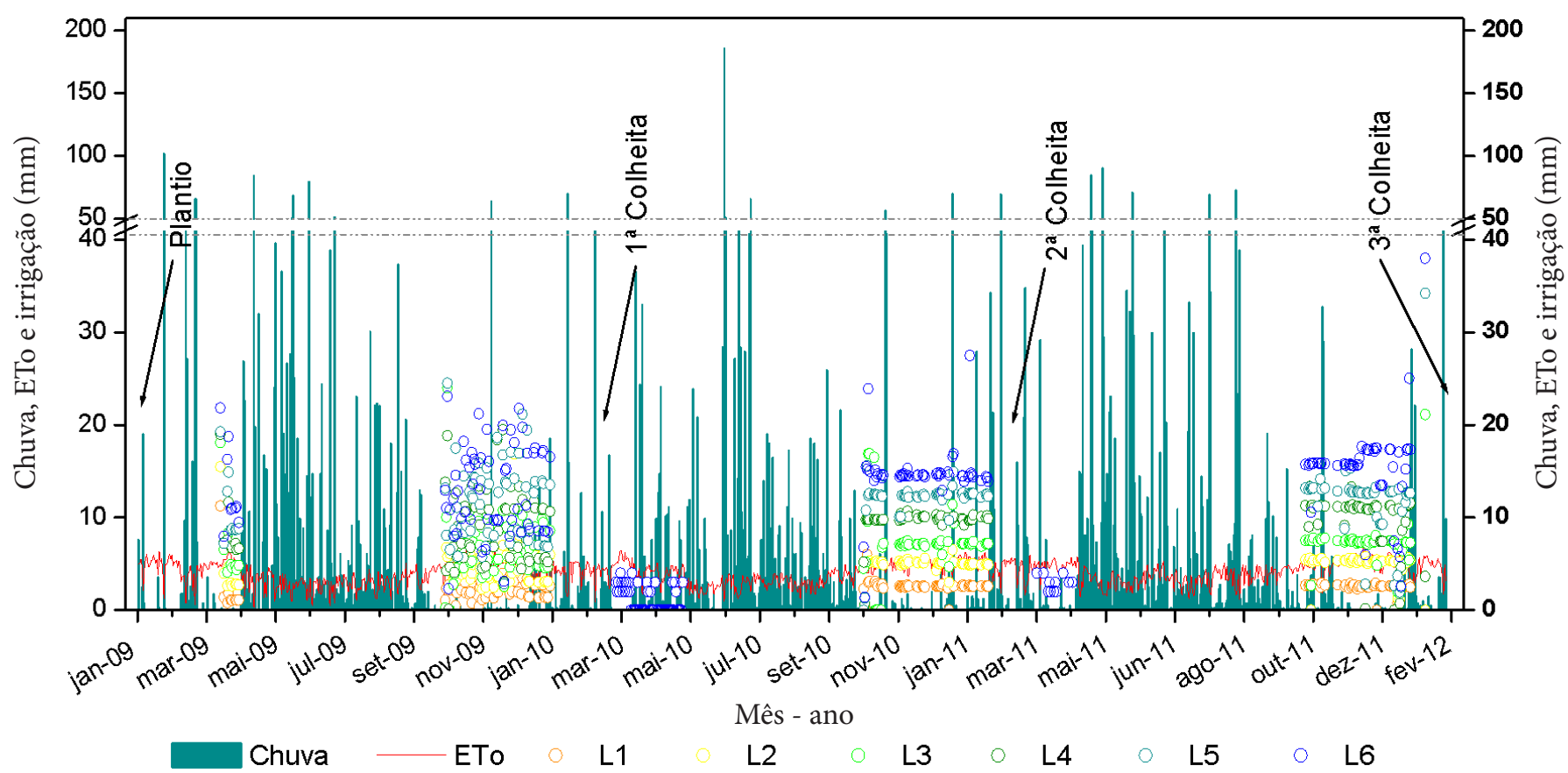

Figura 1. Valores de precipitação pluvial, evapotranspiração de referência ( $\left.E T_{0}\right)$ e lâminas de irrigação ( $L 0, L 1, L 2, L 3$, L4, L5 e L6), durante três ciclos de cultivo da cana-de-açúcar, no período de janeiro de 2009 a fevereiro de 2012, na região de Rio Largo, $\mathrm{AL}$ 
probabilidade de ocorrer déficit hídrico em cultivos de sequeiro durante os meses de outubro a fevereiro Carvalho et al. (2013b), concluíram, com base na estimativa de probabilidade de $80 \%$, que a estação chuvosa na região de Rio Largo, $\mathrm{AL}$, se inicia em abril e termina em outubro. Ocasionalmente e mesmo durante a estação chuvosa, há períodos sem chuva superiores a sete dias, os quais provocam pequenos estresses hídricos nas plantas (Carvalho et al., 2013a).

A evapotranspiração de referência $\left(\mathrm{ET}_{0}\right)$ totalizou 1.616 $\mathrm{mm}$ no $1^{\circ}$ ciclo de cultivo, com mínima de $0,3 \mathrm{~mm} \mathrm{~d}^{-1}(22 \mathrm{de}$ abril de 2009), máxima de $6,6 \mathrm{~mm} \mathrm{~d}^{-1}$ (23 de outubro de 2009) e média de $4,0 \mathrm{~mm} \mathrm{~d}^{-1}$. No $2^{\circ}$ ciclo de cultivo a $\mathrm{ET}_{0}$ acumulada foi de $1.481 \mathrm{~mm}$, com mínima de $1,0 \mathrm{~mm} \mathrm{~d}^{-1}$ (04 de junho de 2010 , quando ocorreu chuva de $186 \mathrm{~mm}$ ), máxima de $6,5 \mathrm{~mm}$ $\mathrm{d}^{-1}$ (06 de março de 2010) e média de $4,0 \mathrm{~mm} \mathrm{~d}^{-1}$. No $3^{\circ}$ ciclo de produção da cultura a $\mathrm{ET}_{0}$ acumulada alcançou $1.493 \mathrm{~mm}$ com mínima de $1,1 \mathrm{~mm} \mathrm{~d}^{-1}$ (25 de agosto de 2011 ), máxima de $7,2 \mathrm{~mm} \mathrm{~d}^{-1}$ (11 de dezembro de 2011) e média de 4,2 $\mathrm{mm} \mathrm{d}^{-1}$. Os maiores valores de $\mathrm{ET}_{0}$ foram observados entre setembro e fevereiro e os menores entre abril e agosto. Portanto, a demanda atmosférica é menor no período em que há maior precipitação pluvial devido à diminuição da radiação solar que é atenuada pela alta nebulosidade.

A irrigação foi iniciada na $1^{\text {a }}$ fase de desenvolvimento da cultura (mês de março), em decorrência da falta de chuvas neste período para suprir a demanda hídrica das plantas. As lâminas de irrigação diferenciadas foram aplicadas no período compreendido entre o final da fase vegetativa e o início da fase final (maturação), ou seja, entre o mês de outubro e o mês de janeiro. Neste período ocorreram redução do armazenamento de água no solo e aumento da evapotranspiração. Os valores médios diários das lâminas de irrigação aplicadas foram 2,2, 4,4, 6,0, 9,0, 10,7 e 12,9 mm em L1, L2, L3, L4, L5 e L6, respectivamente.

No primeiro dia de cada ciclo de produção da cultura, estabelecidos pelo primeiro dia após o plantio ou após o corte, o valor do ARM a $30 \mathrm{~cm}$ de profundidade foi igual ao da capacidade de armazenamento de água disponível ( $\mathrm{CAD}=$ $29,1 \mathrm{~mm}$ ), em razão da irrigação aplicada nessas datas. $\mathrm{Na}$ fase inicial do primeiro ano de cultivo (1-60 DAP), as chuvas foram suficientes para manter a umidade do solo acima do limite de água facilmente disponível (AFD) (Figura 2). Entretanto, no início da fase vegetativa não ocorreram chuvas e o ARM começou a diminuir de modo que aos 69 dias após o plantio (DAP) atingiu o valor de $11,9 \mathrm{~mm}$, sendo menor que a AFD $(12,2 \mathrm{~mm})$. Com isto, houve necessidade de irrigação para suprir o déficit hídrico nos tratamentos irrigados. A partir dos 89 DAP iniciou-se o período chuvoso e o ARM aumentou, permanecendo próximo à CAD durante o restante da fase de crescimento.
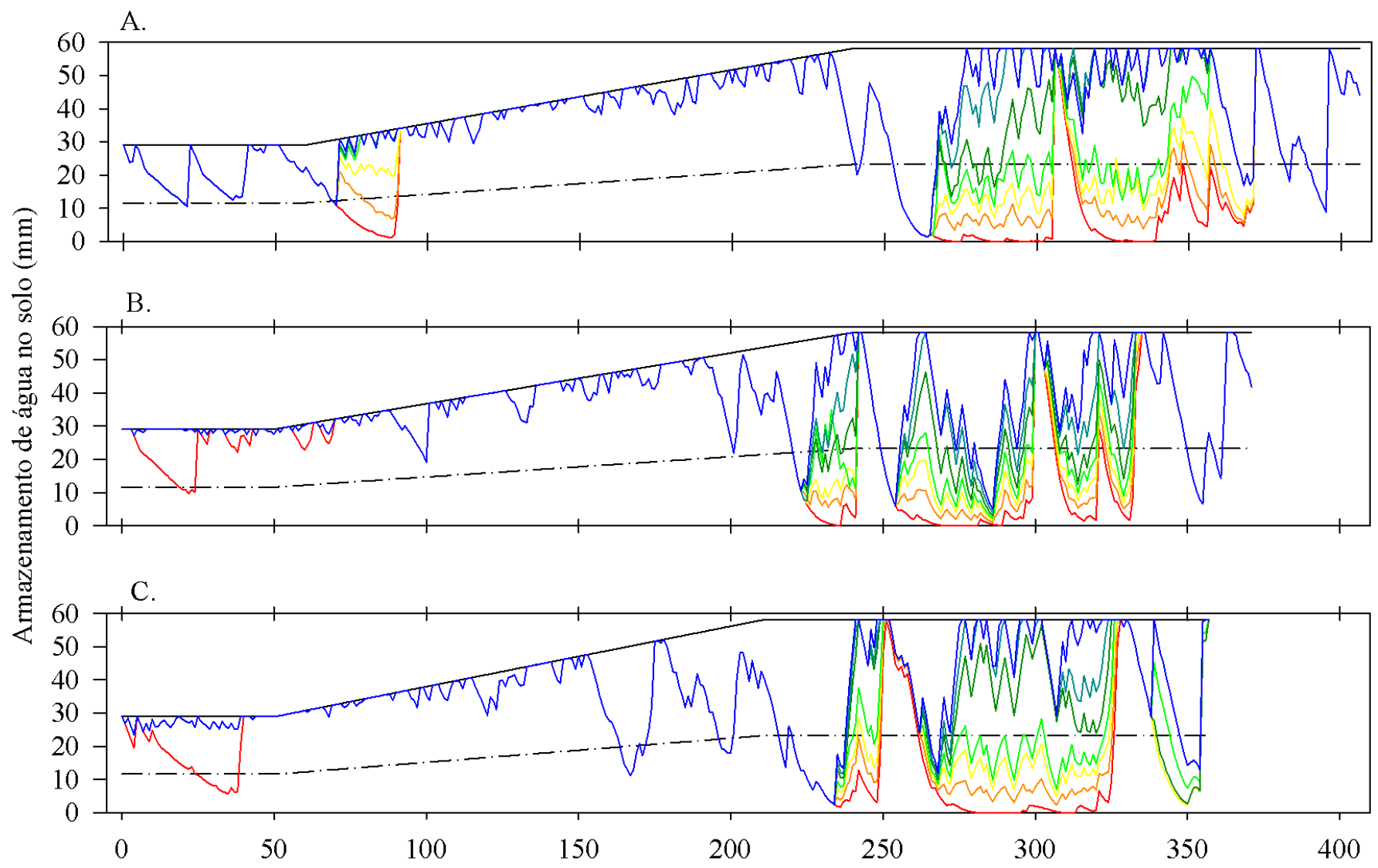

$-\mathrm{CAD}$

Dias após o plantio/colheita

-.-.-AFD

ARM para:

L1

L2

L3

L4

L5

L6

Figura 2. Valores diários da capacidade de armazenamento de água disponível (CAD), limite de água facilmente disponível (AFD) e armazenamento de água no solo (ARM) para tratamentos com diferentes lâminas de irrigação (LO, L1, L2, L3, L4, L5 e L6) em cultivos da cana-de-açúcar anos de 2009 (A), 2010 (B) e 2011 (C), na região de Rio Largo, AL 
Nas fases vegetativa (61-240 DAP no $1^{\circ}$ ciclo, 51-240 DAC no $2^{\circ}$ ciclo e 51-210 DAC no $3^{\circ}$ ciclo), o ARM esteve próximo da $\mathrm{CAD}$ na maior parte deste período porém em algumas ocasiões o ARM foi menor que a AFD. A partir dos 266 DAP do $1^{\circ}$ ciclo e dos 224 e 235 DAC dos $2^{\circ}$ e $3^{\circ}$ ciclos de cultivo, respectivamente, observou-se que o armazenamento de água no solo esteve abaixo do limite crítico de umidade (ARM < AFD) em todos os tratamentos. Este fato ocorreu em decorrência da baixa frequência de chuvas neste período e também porque não havia começado a irrigação; desta forma, a cultura esteve em condições de déficit de água e foi positivo iniciar a aplicação das lâminas de irrigação dos tratamentos.

Nas fases intermediárias (241-346 DAP no $1^{\circ}$ ciclo, 241-320 DAC no $2^{\circ}$ ciclo e 211-307 DAC no $3^{\circ}$ ciclo), quando a cultura estava em crescimento máximo foi aplicada a maior parte das lâminas de irrigação dos tratamentos, fase na qual se observou que nos tratamentos com as menores lâminas de irrigação o déficit de água foi considerável.

$\mathrm{Na}$ fase final a irrigação foi suspensa aos 31, 38 e 27 dias antes da colheita nos $1^{\circ}, 2^{\circ}$ e $3^{\circ}$ anos de cultivo, respectivamente. Este procedimento foi adotado porque a proximidade da colheita exige a imposição de estresse hídrico para induzir as plantas à maturação, ou seja, diminuir o crescimento vegetativo e intensificar o processo de acúmulo de açúcar (Teodoro, 2011).

Observou-se que, ao longo do desenvolvimento da cultura e em função do tempo, a área de solo exposta foi reduzindo e diminuindo a evaporação direta (Abranches \& Bolonhezi, 2011), ou seja, a evapotranspiração passou a ser representada em maior parte pela transpiração haja vista a área foliar se tornou mais importante para a transferência de água para a atmosfera. Conforme a Figura 3, a $\mathrm{ET}_{\mathrm{r}}$ média diária da fase inicial foi de $1,51 \mathrm{~mm} \mathrm{~d}^{-1}$ no $1^{\circ}$ ciclo, $1,77 \mathrm{~mm} \mathrm{~d}^{-1}$ no $2^{\circ}$ e 1,76 $\mathrm{mm} \mathrm{d}^{-1}$ no $3^{\circ}$ ciclo nos tratamentos com irrigação; esses valores estão 30,15 e $20 \%$, respectivamente, abaixo da demanda máxima da cultura (ET, que foi de 2,16, 2,09 e 2,19 $\mathrm{mm} \mathrm{d}^{-1}$ nos $1^{\circ}, 2^{\circ}$ e $3^{\circ}$ ciclos, respectivamente). Esses resultados mostram que as lâminas aplicadas nos tratamentos durante a fase inicial foram suficientes para suprir a transpiração das plantas; no sequeiro a média evapotranspirada foi de $1,51 \mathrm{~mm} \mathrm{~d}^{-1}$ no $1^{\circ}$ ciclo, $1,53 \mathrm{~mm} \mathrm{~d}^{-1}$ no $2^{\circ}$ e $1,58 \mathrm{~mm} \mathrm{~d}^{-1}$ no $3^{\circ}$ ciclo, sendo 30 , 27 e $28 \%$ menor que a $\mathrm{ET}_{\mathrm{c}}$, respectivamente.

Como o sistema de irrigação usado foi o gotejamento, não se recomenda a aplicação de lâmina diária superior a $2 \mathrm{~mm}$ durante a fase inicial (até 60 DAP ou DAC), em função da limitação da $\mathrm{ET}_{\mathrm{r}}$. A aplicação de lâminas superiores resulta em perda de água por drenagem devido à diferença entre o ARM e a CAD. Além de que a diminuição da frequência de aplicação, ou seja, o aumento dos intervalos de tempo entre irrigações para períodos superiores a 3 dias, causa déficit de água no solo pois nos dias sem irrigação existe uma demanda para suprir a $\mathrm{ET}_{\mathrm{r}}$. Portanto, caso seja necessário aumentar o turno de rega recomenda-se aplicar uma lâmina de $6 \mathrm{~mm}$ a cada 3 dias para

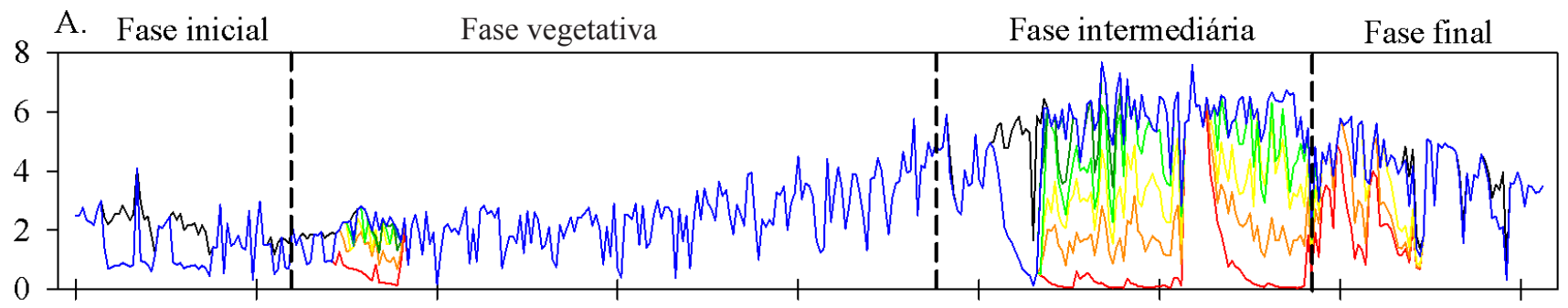

B.
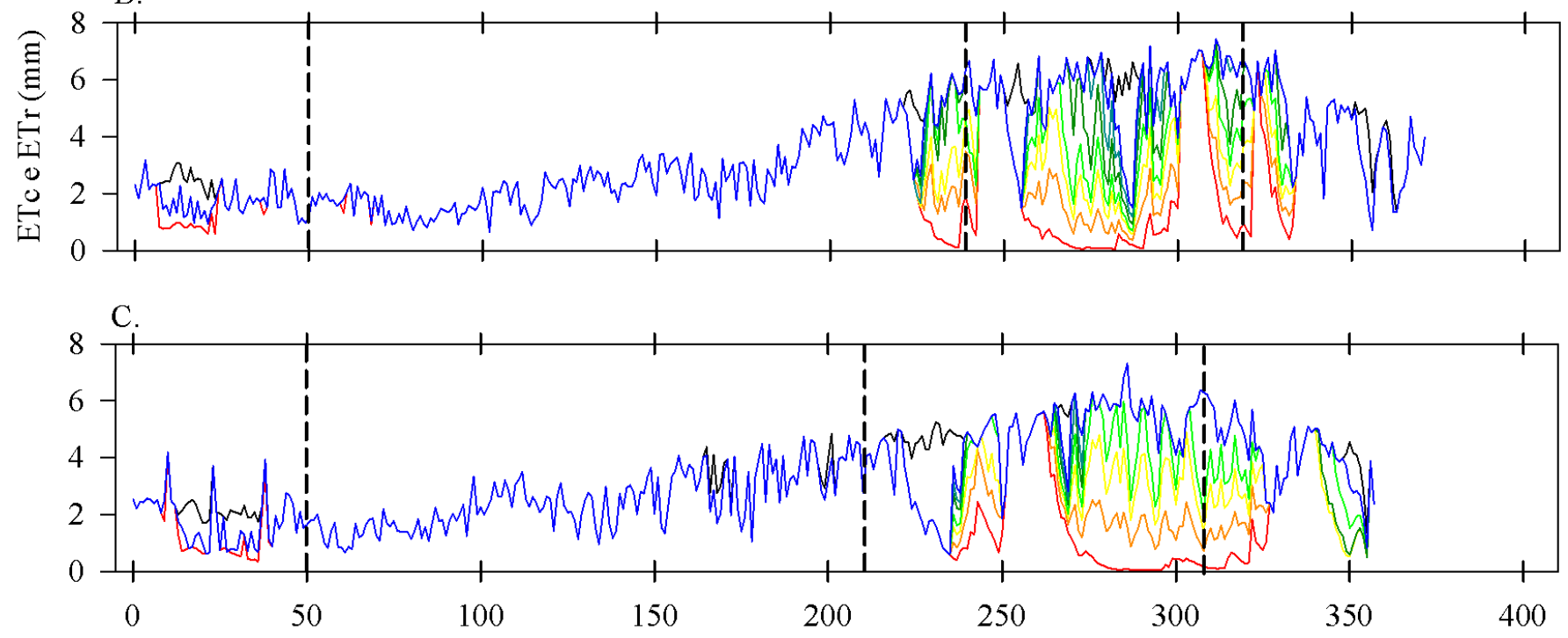

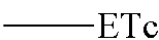

ETr para:

L0

Dias após o plantio/colheita

Figura 3. Valores diários da evapotranspiração da cultura (ETc) e evapotranspiração real da cana-de-açúcar (ETr) para tratamentos com lâminas de irrigação diferenciadas (LO, L1, L2, L3, L4, L5 e L6) nas safras de 2009/2010 (A), 2010/2011 (B) e 2011/2012 (C), na região de Rio Largo, AL 
manter a umidade do solo próximo à capacidade de campo durante a fase inicial de crescimento, sendo o ARM igual ou muito próximo ao valor da CAD.

Entre os 71 e 87 DAP houve um período seco e, com isto a necessidade de irrigação até o retorno das chuvas. Com o início do período chuvoso na fase de crescimento ocorreu reposição da umidade do solo, de forma que a $\mathrm{ET}_{\mathrm{r}}$ de todos os tratamentos se igualou à $\mathrm{ET}_{\mathrm{c}}$.

Com a redução das chuvas o armazenamento de água no solo diminuiu e o consumo diário de água pela cultura não foi totalmente suprido, reduzindo os valores de $\mathrm{ET}_{\mathrm{r}}$. Assim, aos 266 DAP no $1^{\circ}$ ciclo e aos 224 e 235 DAC nos $2^{\circ}$ e $3^{\circ}$ ciclos de cultivo, respectivamente, foi preciso iniciar a irrigação diferenciada de acordo com os respectivos tratamentos. $\mathrm{Na}$ fase vegetativa do $1^{\circ}$ ciclo, a $\mathrm{ET}_{\mathrm{r}}$ no tratamento de sequeiro variou de 0,13 a $5,76 \mathrm{~mm} \mathrm{~d}^{-1}$ e nos demais tratamentos de 0,15 a $5,76 \mathrm{~mm} \mathrm{~d}^{-1}$. No $2^{\circ}$ ciclo a $\mathrm{ET}_{\mathrm{r}}$ variou de 0,11 a $5,34 \mathrm{~mm}$ $\mathrm{d}^{-1}$ no tratamento de sequeiro, de 0,66 a $5,34 \mathrm{~mm} \mathrm{~d}^{-1} \mathrm{em} \mathrm{L1} \mathrm{e}$ L2, de 0,66 a 6,21 $\mathrm{mm} \mathrm{d}^{-1}$ em L3, e de 0,66 a 6,67 $\mathrm{mm} \mathrm{d}^{-1} \mathrm{em}$ L4, L5 e L6. No $3^{\circ}$ ciclo a ET variou de 0,65 a $4,77 \mathrm{~mm} \mathrm{~d}^{-1} \mathrm{em}$ todos os tratamentos.

$\mathrm{Na}$ fase intermediária a $\mathrm{ET}_{\mathrm{r}}$ foi influenciada em maior proporção pela transpiração porque o dossel da cultura já estava completamente estabelecido. Como nesta fase houve baixa precipitação pluvial e foi aplicada a maior parte das lâminas de irrigação diferenciadas, pode-se observar que o déficit de água afetou bastante a transpiração da cultura em que, para as menores lâminas, houve redução da $\mathrm{ET}_{\mathrm{r}}$. $\mathrm{A} \mathrm{ET}_{\mathrm{r}}$ dos tratamentos L6 e L5 foi inferior à $\mathrm{ET}_{c}$ em alguns dias, em virtude das interrupções na irrigação causadas por problemas operacionais. A média de $\mathrm{ET}_{\mathrm{r}}$ na fase intermediária dos três ciclos foi de 1,73, 2,59, 3,44, 4,10, 4,74, 4,96 e 5,04 $\mathrm{mm} \mathrm{d}^{-1}$ para os tratamentos com L0, L1, L2, L3, L4, L5 e L6, respectivamente. A ET média na fase intermediária nos $1^{\circ}, 2^{\circ}$ e $3^{\circ}$ ciclos de cultivo, foi de $5,48,6,01$ e $5,10 \mathrm{~mm} \mathrm{~d}^{-1}$, com média geral de $5,53 \mathrm{~mm} \mathrm{~d}^{-1}$. Considerando a média geral da $\mathrm{ET}_{\mathrm{c}}$ na fase intermediária dos três ciclos $\left(5,53 \mathrm{~mm} \mathrm{~d}^{-1}\right)$, se não houvesse irrigação nesta fase o consumo de água pela cultura durante o período de 6 dias faria o ARM decrescer da CAD $(58,2 \mathrm{~mm})$ ao limite da AFD (23,3 mm).

Os valores de $\mathrm{ET}_{\mathrm{r}}$ e de $\mathrm{ET}_{\mathrm{c}}$ observados em cada tratamento durante a fase intermediária, estão sujeitos às variações anuais; apesar disto e devido à baixa amplitude dessas variações, os mesmos podem ser tomados como referência para se fazer o manejo de irrigação na cana-de-açúcar. Como é comum, entre os produtores do Nordeste brasileiro, priorizar as áreas irrigadas por gotejamento para colher, no final da safra, a fase intermediária de desenvolvimento da cultura coincide com o período seco e a necessidade hídrica da planta é semelhante à encontrada neste experimento.

Na última fase do ciclo de produção da cultura a $\mathrm{ET}_{\mathrm{r}}$ tendeu a diminuir em decorrência da suspensão da irrigação, do início da senescência das plantas e da moderada magnitude e frequência das chuvas. Os valores de $\mathrm{ET}_{\mathrm{r}}$ foram 3,47, 3,98 e 2,37 $\mathrm{mm} \mathrm{d}^{-1}$ para todos os tratamentos na véspera da colheita nos $1^{\circ}, 2^{\circ}$ e $3^{\circ}$ ciclos de produção, respectivamente; enfim e devido à suspensão da irrigação, todos os tratamentos se mantiveram nas mesmas condições de umidade.

Silva et al. (2012b) determinaram o requerimento hídrico da cana-soca irrigada, variedade RB92579, cultivada na região semiárida do Submédio do Vale do São Francisco (Juazeiro, BA). Os autores observaram que na fase de brotação e estabelecimento da cultura, a ET oscilou em torno de 3,0 $\mathrm{mm} \mathrm{d}^{-1}$ no entanto, na fase de estabelecimento e perfilhamento o valor médio da ET aumentou para $3,8 \mathrm{~mm} \mathrm{~d}^{-1}$, enquanto na fase vegetativa máxima os valores da evapotranspiração da cultura atingiram, em média, 5,1 $\mathrm{mm} \mathrm{d}^{-1}$, destacando-se como o mais elevado do ciclo produtivo da cana-de-açúcar. Finalmente, na fase de maturação da cultura em que ocorreu a suspensão da irrigação para promover o acúmulo de sacarose nos colmos, os valores da $\mathrm{ET}_{\mathrm{c}}$ decresceram para o valor médio de $3,1 \mathrm{~mm} \mathrm{~d}^{-1}$.

A partir de dados obtidos em uma revisão de literatura, Santos (2005) comparou a evapotranspiração da cana-deaçúcar em diferentes regiões do mundo observando que, a depender da variedade, do local de cultivo e do sistema de produção, os valores mínimos, médios e máximos de $\mathrm{ET}_{\text {c }}$ variam de 0,52 a $4,10,2,33$ a 5,70 e de 3,70 a $8,60 \mathrm{~mm} \mathrm{~d}^{-1}$, respectivamente. Thompson \& Boyce (1967) realizaram medições em lisímetros cultivados com cana-de-açúcar em condições climáticas de Pongola, na África do Sul, obtiveram valor máximo de $\mathrm{ET}_{\mathrm{c}}$ igual a $6,8 \mathrm{~mm} \mathrm{~d}^{-1}$.

Os $1^{\circ}, 2^{\circ}$ e $3^{\circ}$ ciclos de cultivo, com duração de 406, 371 e 357 dias, totalizaram 2.208, 1.952 e $1.915 \mathrm{~mm}$ de precipitação pluvial, respectivamente; entretanto, nos $1^{\circ}, 2^{\circ}$ e $3^{\circ}$ ciclos a precipitação efetiva variou de 705 a $834 \mathrm{~mm}$, de 611 a 841 $\mathrm{mm}$ e de 609 a $762 \mathrm{~mm}$ no tratamento L6 e no sequeiro, respectivamente (Tabela 1). As lâminas de irrigação variaram de 157 a $873 \mathrm{~mm}$, de 127 a $734 \mathrm{~mm}$ e de 117 a $707 \mathrm{~mm}$ entre L1 a L6, nos $1^{\circ}, 2^{\circ}$ e $3^{\circ}$ ciclos, respectivamente. Assim, a lâmina total

Tabela1. Valores de precipitação pluvial, irrigação, ET, e ER para tratamentos com lâminas de irrigação em três ciclos de cultivo da cana-de-açúcar na região de Rio Largo, Ár

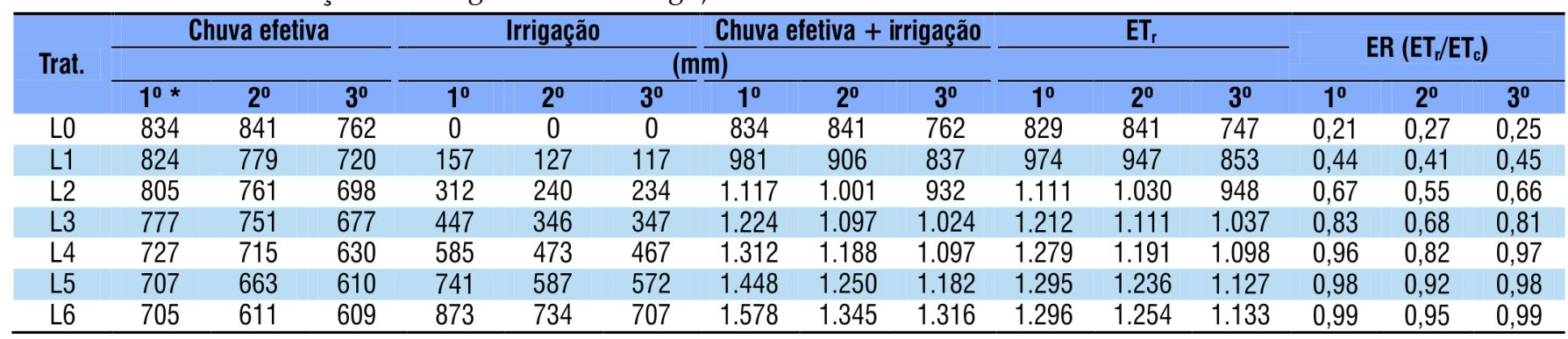

* Número correspondente ao ciclo de produção da cultura, em que 10: cana-planta (2009-2010, 406 dias com chuva total de 2.208 mm); $2^{0}$ : primeira soca (2010-2011, 371 dias com chuva total de $1.952 \mathrm{~mm}$ ); $3^{0}$ : segunda soca (2011-2012, 357 dias com chuva total de $1.915 \mathrm{~mm}$ ); Trat. - tratamento 
efetiva aplicada nos tratamentos irrigados esteve no intervalo de 981 a $1.578 \mathrm{~mm}$, de 906 a $1.345 \mathrm{~mm}$ e de 837 a $1.316 \mathrm{~mm}$ em L1 a L6, nos $1^{\circ}, 2^{\circ}$ e $3^{\circ}$ ciclos, respectivamente.

A evapotranspiração máxima da cultura $\left(\mathrm{ET}_{\mathrm{c}}\right)$ totalizou $1.397,1.329$ e $1.223 \mathrm{~mm}$ mas a $\mathrm{ET}_{\mathrm{r}}$ variou de 929 a $1.296 \mathrm{~mm}$, de 841 a $1.254 \mathrm{~mm}$ e de 747 a $1.133 \mathrm{~mm}$ no sequeiro e em L6, nos $1^{\circ}, 2^{\circ}$ e $3^{\circ}$ ciclos, respectivamente. Esses valores totais indicam que o tratamento de sequeiro teve o índice de satisfação das necessidades de água da cultura (ISNA) igual a 0,56, 0,60 e 0,61 nos $1^{\circ}, 2^{\circ}$ e $3^{\circ}$ ciclos, respectivamente. Portanto, conforme a classificação descrita por Silva et al. (2013), o cultivo de sequeiro esteve exposto a médio risco climático, considerando apenas os três anos de medições. A evapotranspiração relativa (ER) de todos os tratamentos, calculada apenas para o período em que houve irrigação, variou de 0,21 a 0,99, de 0,27 a 0,95 e de 0,25 a 0,99 entre L0 a L6, nos $1^{\circ}, 2^{\circ}$ e $3^{\circ}$ ciclos, respectivamente. Como as lâminas de irrigação do tratamento L1 e L2 somadas à precipitação efetiva do período seco nos três ciclos de cultivo não atenderam à demanda hídrica máxima da cultura, a ER esteve abaixo do valor aceitável $(0,75)$ para cultivos irrigados, conforme recomendado por Roerink et al. (1997).

Oliveira et al. (2012) observaram que mais de 73\% da área do Nordeste estão classificados como região de alto risco climático para o cultivo da cana-de-açúcar, devido às irregularidades na distribuição de chuvas ao longo do ano porém a região próxima ao litoral possui baixo e médio risco climático para o cultivo em quase todos os meses do ano. Referida classificação climática pode, em função do suprimento hídrico, variar em uma mesma região; assim, a evapotranspiração real pode ser menor que a evapotranspiração máxima da cultura tanto se houver diminuição da reposição hídrica pelas chuvas quanto aumento da temperatura do ar.

Teodoro et al. (2009) mediram, avaliando a cultura da cana-de-açúcar durante 420 dias em regime de sequeiro, precipitação pluvial de $1.825 \mathrm{~mm}$ porém do $3^{\circ}$ decêndio de abril ao $1^{\circ}$ decêndio de agosto de 2006 , ocorreu excesso hídrico de $837 \mathrm{~mm}$. Como a evapotranspiração máxima da cultura foi estimada em $2.068 \mathrm{~mm}$, houve um déficit hídrico que poderia ser suprido quase que totalmente se o excedente das chuvas fosse armazenado e utilizado para irrigação, Abreu et al. (2013).

Silva et al. (2012b) encontraram requerimento hídrico total de $1.710 \mathrm{~mm}$ em cana-soca irrigada, variedade RB92579, cultivada na região semiárida do Submédio do Vale do São Francisco, durante 357 dias. Os autores observaram que o suprimento hídrico foi satisfatório visto que a ER foi de 0,92 (Silva et al., 2011). Almeida et al. (2008) mediram a evapotranspiração acumulada de $1.584 \mathrm{~mm}$ em 12 meses para a mesma variedade (RB92579) cujos dados se referem ao segundo ciclo de cultivo sob as condições irrigadas da região de Rio Largo, AL.

Lyra et al. (2007b) encontraram, pesquisando a $\mathrm{ET}_{\mathrm{c}}$ da canaplanta em cultivo de sequeiro no período de outubro de 2000 a janeiro de 2002, na região de Maceió, AL, ET total de 1.180 $\mathrm{mm}$ em 450 dias com uso do $\mathrm{K}_{\mathrm{c}}$ "dual”. Para a mesma época de cultivo porém utilizando o $\mathrm{K}_{\mathrm{c}}$ conjugado para evaporação e transpiração, Lyra et al. (2007a) determinaram a ET total de $2.050 \mathrm{~mm}$, sendo $74 \%$ superiores ao valor obtido com o $\mathrm{K}_{\mathrm{c}}$ "dual". Mencionada diferença se deve ao fato de que o método "dual" é mais eficiente no que diz respeito à contabilização da evaporação direta de água no solo, particularmente em cultivos de sequeiro e irrigado por gotejamento nos quais a evaporação exerce pouca influência na $\mathrm{ET}_{\mathrm{r}}$ indicando que o procedimento de estimativa da $\mathrm{ET}_{\mathrm{r}}$ também deve ser escolhido em função do método de irrigação a ser utilizado. Além disto, para o manejo adequado da irrigação baseado na $\mathrm{ET}_{\mathrm{r}}$, os coeficientes da cultura devem ser ajustados às condições climáticas locais em cada fase de desenvolvimento, principalmente na fase inicial em que há predominância da evaporação direta da água do solo.

\section{Conclusões}

1. A evapotranspiração real varia conforme os valores diferenciados das lâminas de irrigação aplicadas à cultura da cana-de-açúcar. Como em sistemas de irrigação por gotejamento a contribuição da evaporação é menor, sobremaneira após o estabelecimento do dossel da cultura, a transpiração real diminui com a redução da lâmina aplicada.

2. Com base no índice de satisfação das necessidades de água da cultura o cultivo da cana-de-açúcar em regime de sequeiro na região de Rio Largo, Alagoas, está exposto a médio risco climático, conforme enquadramento proposto na literatura.

\section{Literatura Citada}

Abranches, J. L.; Bolonhezi, A. C. Desenvolvimento inicial de variedades e clones de cana-de-açúcar em Latossolo Vermelho Distrófico, Aparecida do Taboado-MS. Revista Brasileira de Ciências Agrárias, v.6, p.369-375, 2011. http://dx.doi. org/10.5039/agraria.v6i3a716

Abreu, M. L.; Silva, M. A.; Teodoro, I.; Holanda, L. A.; Sampaio Neto, G. D. Crescimento e produtividade de cana-de-açúcar em função da disponibilidade hídrica dos Tabuleiros Costeiros de Alagoas. Bragantia, v.72, p.262-270, 2013. http://dx.doi. org/10.1590/brag.2013.028

Allen, R. G.; Pereira, L. S.; Smith, M.; Raes, D.; Wright, J. L. FAO-56 Dual crop coefficient method for estimatingevaporation from soil and application extensions. Journal of Irrigation and Drainage Engineering, v.131, p.1-13, 2005. http://dx.doi.org/10.1061/ (ASCE)0733-9437(2005)131:1(2)

Almeida, A. C. S.; Souza, J. L.; Teodoro, I.; Barbosa, G. V. S.; Moura Filho, G.; Ferreira Júnior, R. A. Desenvolvimento vegetativo e produção de variedades de cana-de-açúcar em relação à disponibilidade hídrica e unidades térmicas. Ciência e Agrotecnologia, v.32, p.1441-1448, 2008. http://dx.doi. org/10.1590/S1413-70542008000500013

Boas, R. C. V.; Pereira,G. M.; Reis, R. P.; Lima Júnior, J. A.; Consoni, R. Viabilidade econômica do uso do sistema de irrigação por gotejamento na cultura da cebola. Ciência e Agrotecnologia, v.35, p.781-788, 2011. http://dx.doi.org/10.1590/S141370542011000400018

Carvalho, A. L.; Souza, J. L.; Lyra, G. B.; Porfirio, A. C. S. Ocorrência de períodos secos na região de Rio Largo, Alagoas. Revista Brasileira de Meteorologia, v.28, p.173-180, 2013a. http://dx.doi. org/10.1590/S0102-77862013000200006 
Carvalho, A. L.; Souza, J. L.; Lyra, G. B.; Silva, E. C. Estação chuvosa e de cultivo para a região de Rio Largo, Alagoas baseada em métodos diretos e sua relação com o El Niño - Oscilação Sul. Revista Brasileira de Meteorologia, v.28, p.192-198, 2013 b. http://dx.doi.org/10.1590/S0102-77862013000200008

Carvalho, O. M: Classificação e caracterização físico-hídrica de solos de Rio-largo,cultivados com cana-de-açúcar. Rio Largo: Universidade Federal de Alagoas, 2003. 74p. Dissertação Mestrado

Dantas Neto, J.; Figueredo, J. L. C.; Farias, C. H. de A.; Azevedo, H. M. de; Azevedo, C. A. V. de. Resposta da cana-de-açúcar, primeira soca, a níveis de irrigação e adubação de cobertura. Revista Brasileira de Engenharia Agrícola e Ambiental, v.10, p.283-288, 2006. http://dx.doi.org/10.1590/S1415-43662006000200006

Lyra, G. B.; Pereira, A. R.; Lyra, G. B.; Sediyama, G. C.; Maia, S. M. F. Evapotranspiração da cultura de cana-de-açúcar na região de Tabuleiros Costeiros do estado de Alagoas: coeficiente da cultura "dual" padrão boletim FAO-56. Revista da STAB, v.25, p.44-51, $2007 \mathrm{a}$.

Lyra, G. B.; Sediyama, G. C.; Lyra, G. B.; Pereira, A. R.; Souza, E. F. Evapotranspiração da Cultura de Cana de Açúcar na Região de Tabuleiros Costeiros do Estado de Alagoas: Coeficiente da Cultura "Único" Padrão Boletim FAO-56 Kc_unico2007. Revista da STAB, v.25, p.40-43, 2007b.

Martins, C. A. S.; Reis, E. F.; Nogueira, N. O. Análise do desempenho da irrigação por microspray na cultura do café conilon. Enciclopédia Biosfera, v.7, p.1-13, 2011.

Oliveira, S. D.; Silva, V. P. R.; Santos, C. A. C.; Silva, M. T.; Sousa, E. P. Os Impactos das Alterações Climáticas na Cana-de-açúcar Cultivada em Sistema de Sequeiro na Região Nordeste do Brasil. Revista Brasileira de Geografia Física, v.1, p.170-184, 2012.

Parkes, M.; Yao, W. W.; Ma, X. Y.; Li, J. Simulation of point source wetting pattern of subsurface drip irrigation. Irrigation Science, v.29, p.331-339, 2010.

Pereira, A. R.; Angelocci, L. R.; Sentelhas, P. C. Agrometeorologia (Fundamentos e aplicações práticas). Guaíba: Agropecuária, 2002. 478p.

Roerink, G. J.; Bastiaanssen, W. G. M.; Chambouleyron, J.; Menenti, M. Relating crop water consumption to irrigation water supply by remote sensing. Water Resources Management, v.11, p.445-465, 1997. http://dx.doi.org/10.1023/A:1007982411718

Santos, M. A. L. Irrigação suplementar da cana-de-açúcar (Saccharum spp.): Um modelo de análise de decisão para o Estado de Alagoas. Piracicaba: ESALQ/USP, 2005. 101p. Tese Doutorado
Silva, M. A.; Arantes, M. T.; Rhein, A. F. L.; Gava, G. J. C.; Kolln, O. T. Potencial produtivo da cana-de-açúcar sob irrigação por gotejamento em função de variedades e ciclos. Revista Brasileira de Engenharia Agrícola e Ambiental, v.18, p.241-249, 2014. http://dx.doi.org/10.1590/S1415-43662014000300001

Silva, S.; Teodoro, I.; Lyra, G. B.; Souza, J. L.; Dantas Neto, J. Adaptação do método de Kc "dual" (FAO-56) para a cana-deaçúcar irrigada por gotejamento. ABEAS, v.27, p.87-93, 2012a. http://dx.doi.org/10.12722/0101-756X.v27n02a02

Silva, T. G. F.; Moura, M. S. B.; Zolnier, S.; Soares, J. M.; Vieira, V. J. S.; Gomes Júnior, W. F. Demanda hídrica e eficiência do uso de água da cana-de-açúcar irrigada no semiárido brasileiro. Revista Brasileira de Engenharia Agrícola e Ambiental, v.15, p.1257-1265, 2011. http://dx.doi.org/10.1590/S1415-43662011001200007

Silva, T. G. F.; Moura, M. S. B.; Zolnier, S.; Soares, J. M.; Vieira, V. J. S.; Gomes Júnior, W. F. Requerimento hídrico e coeficiente de cultura da cana-de-açúcar irrigada no semiárido brasileiro. Revista Brasileira de Engenharia Agrícola e Ambiental, v.16, p.64-71, 2012b.

Silva, V. de P. R. da; Oliveira, S. D.; Santos, C. A. C.; Silva, M. T. Risco climático da cana-de-açúcar cultivada na região Nordeste do Brasil. Revista Brasileira de Engenharia Agrícola e Ambiental, v.17, p.180-189, 2013. http://dx.doi.org/10.1590/S141543662013000200009

Souza, J. L.; Moura Filho, G.; Lyra, R. F. F.; Teodoro, I.; Santos, E. A.; Silva, J. L.; Silva, P. R. T.; Cardim, A. H.; Amorim, E. C. Análise da precipitação pluvial e temperatura do ar na região do Tabuleiro Costeiro de Maceió, AL, período 1972-2001. Revista Brasileira de Agrometeorologia, v.12, p.131-141, 2004.

Teodoro, I. Respostas técnico-econômicas da cana-de-açúcar a níveis de irrigação e adubação nitrogenada. Campina Grande: UFCG, 2011. 100p. Tese Doutorado

Teodoro, I.; Dantas Neto, J.; Souza, J. L.; Lyra, G. B.; Brito, K. S.; Sá, L. A.; Santos, M. A. L.; Sarmento, P. L. V. S. Isoquantas de produtividade da cana-de-açúcar em função de níveis de irrigação e adubação nitrogenada. Irriga, v.18, p.387-401, 2013. http:// dx.doi.org/10.15809/irriga.2013v18n3p387

Teodoro, I.; Souza, J. L.; Barbosa, G. V.; Moura Filho, G.; Dantas Neto, J.; Abreu, M. L. Crescimento e Produtividade da Canade-açúcar em Cultivo de Sequeiro nos Tabuleiros Costeiros de Alagoas. STAB, v.27, p.31-34, 2009.

Thompson, G. D.; Boyce, J. P. Daily measurements of potential evapotranspiration from fully canopied sugarcane. Agricultural Meteorology, v.4, p.267-279, 1967. http://dx.doi.org/10.1016/00021571(67)90027-1 